\title{
CONNECTEDNESS AND MINIMAL LENGTH ELEMENTS IN SPACES OF BOUNDED CURVATURE PATHS
}

\author{
JOSÉ AYALA
}

(Received 11 February 2014; first published online 5 June 2014)

2010 Mathematics subject classification: primary 49Q10; secondary 90C47, 51E99, 68R99.

Keywords and phrases: bounded curvature path, Dubins path, path optimisation, connected components.

A bounded curvature path corresponds to a $C^{1}$ and piecewise $C^{2}$ path lying in $\mathbb{R}^{2}$ having its curvature bounded by a positive constant and connecting two elements of the tangent bundle $T \mathbb{R}^{2}$. In this thesis we develop techniques to answer questions about the connectivity of the spaces of bounded curvature paths and to describe the minimal length elements in these spaces. The idea is to analyse spaces of bounded curvature paths and their minimal length elements using geometrical and topological features.

In the 1960s, Dubins [5, page 471] initiated the study of the homotopy classes of bounded curvature paths. He wrote: 'Here we only begin the exploration, raise some questions that we hope will prove stimulating, and invite others to discover the proofs of the definite theorems, proofs that have eluded us.' This thesis provides answers to the key question raised by Lester Dubins, that is, finding sufficient and necessary conditions for two bounded curvature paths to lie in the same homotopy class. This thesis also provides a classification of the minimal length elements of spaces of bounded curvature paths.

Let $\Gamma(n)$ be the space of bounded curvature paths having fixed initial and final directed points $\mathrm{X}, \mathrm{Y} \in T \mathbb{R}^{2}$ and having winding number $n$. (The winding number is defined by 'closing up the paths' using a fixed path from the final to the initial directed points.)

The thesis is divided into two main parts:

- the classification of the minimal length elements in $\Gamma(n)$ for all $n$ and all $\mathrm{X}, \mathrm{Y} \in T \mathbb{R}^{2}$;

- $\quad$ the classification of the homotopy classes in $\Gamma(n)$ for all $n$ and all $\mathrm{x}, \mathrm{Y} \in T \mathbb{R}^{2}$.

\footnotetext{
Thesis submitted to The University of Melbourne in August 2013; degree approved 21 January 2014; supervisors: Hyam Rubinstein, Marcus Brazil and Doreen Thomas.

The author's name is given in the thesis and associated documents as Jose Ayala Hoffmann.

(C) 2014 Australian Mathematical Publishing Association Inc. 0004-9727/2014 \$16.00
} 
An interesting fact is that embedded bounded curvature paths may have different choices of winding numbers, depending on the initial and final points under consideration. By analysing the distances between the points $\mathrm{x}$ and $\mathrm{Y}$ we obtain four distance properties, called proximity conditions. The proximity conditions give qualitative insight into some of the topological features of $\Gamma(n)$; for example the number of homotopy classes in $\Gamma(n)$.

As a useful step towards the classification of minimal length elements in $\Gamma(n)$ for all $n$ and all $\mathrm{X}, \mathrm{Y} \in T \mathbb{R}^{2}$, in Chapter 3 we characterise the bounded curvature paths that are candidates to be of minimum length, via a normalisation process. This is followed by a reduction process performed on bounded curvature paths to obtain the well-known CSC, CCC characterisation as first obtained in [4]. The normalisation and reduction processes are intimately related with the concept of fragmentation of a path and this concept is developed so that the winding number of the paths is not changed.

In Chapter 4 we finish the first part of the thesis by characterising bounded curvature paths of minimal length in $\Gamma(n)$ for all $n$. This is achieved by using some of the ideas developed in Chapter 3.

In the second part of the thesis, we consider a pair of directed points in the tangent bundle of the Euclidean plane and analyse when paths in $\Gamma(n)$ can be made homotopic via a one-parameter family of bounded curvature paths, paying special attention to the proximity conditions satisfied by the points $\mathrm{x}$ and $\mathrm{y}$. We conclude in Chapter 5 that, under certain conditions, there are paths in a planar region $\Omega$ which cannot be continuously deformed to paths which meet the complement of $\Omega$, while preserving the bounded curvature condition. In particular, the region $\Omega$ is compact and no path in $\Omega$ can be homotopic via a one parameter family of bounded curvature paths to a path outside of $\Omega$.

In Chapter 6, we use all the results developed in the previous chapters to prove that some spaces of bounded curvature paths have a single connected component, while other spaces have two connected components.

In Chapter 7, we comment on our future work and the current state of research. We consider a more general class of paths satisfying a condition called the weak bounded curvature property. In particular, we show that the space $\Gamma(n)$ for fixed $\mathrm{X}, \mathrm{Y} \in T \mathbb{R}^{2}$ is contained in the space of paths satisfying the weak curvature property. This is convenient when considering paths on more general surfaces, such as having nonconstant curvature.

Homotopies of bounded curvature paths are key in applications as in [1-3]. For example, in the decline design of an underground mine, the mine is considered as a 3dimensional network where the directed nodes correspond to the surface portal, access points and draw points, and the links correspond to the centrelines of ramps and drives (compare [2]). In addition, the navigability conditions such as the turning radius of vehicles and the gradient of ramps are required. Minimising the cost of the links can be achieved by projecting the problem in the horizontal plane and this is applied in the software DOT (Design Optimisation Tool) developed by the research group in underground mine optimisation at the University of Melbourne. As a consequence of 
the results obtained in Chapter 5, we validated the optimality of the DOT software when performing certain operations required for barrier avoidance.

The study of bounded curvature paths was first begun by Markov [6] in 1889. He considered the problem of finding a curve of minimal length connecting two directed points in the plane, such that the curvature cannot be greater than a prescribed positive number.

The extension of results presented in this thesis for surfaces of constant curvature is a matter of the author's current research. The next step is to extend the theory for nonconstant curvature Riemannian surfaces by addressing the following tasks (in which $\mathbb{M}$ is a nonconstant curvature Riemannian surface):

- $\quad$ characterise the minimal length elements in $\Gamma(n)$ for $n \in \mathbb{Z}$ and $\mathrm{x}, \mathrm{Y} \in T \mathbb{M}$;

- $\quad$ classify the homotopy classes in $\Gamma(n)$ for $n \in \mathbb{Z}$ and $\mathrm{x}, \mathrm{y} \in T \mathbb{M}$.

\section{References}

[1] J. Backer and D. Kirkpatrick, 'Bounded-curvature path normalization', Proc. 18th Canadian Conf. on Computational Geometry (CCCG 2006), Kingston, Ontario, 14-16 August 2006.

[2] M. Brazil, P. A. Grossman, D. A. Thomas, J. H. Rubinstein, D. Lee and N. C. Wormald, 'Constrained path optimisation for underground mine layout', Proc. 2007 Int. Conf. on Applied and Engineering Mathematics (ICAEM'07), London (2007), 856-861.

[3] H. Chitsaz and S. La Valle, 'Time-optimal paths for a Dubins airplane', Proc. 46th IEEE Int. Conf. on Decision and Control (CDC 2007).

[4] L. Dubins, 'On curves of minimal length with constraint on average curvature, and with prescribed initial and terminal positions and tangents', Amer. J. Math. 79 (1957), 139-155.

[5] L. Dubins, 'On plane curves with curvature', Pacific J. Math. 11 (1961), 471-481.

[6] A. A. Markov, 'Some examples of the solution of a special kind of problem on greatest and least quantities', Soobshch. Karkovsk. Mat. Obshch. 1 (1887), 250-276 (in Russian).

JOSÉ AYALA, Universidad Arturo Prat, Iquique, Chile

e-mail: jayalhoff@gmail.com 